\title{
Users' Experience in Digital Architectural Design: Combining Qualitative Research Methods with a Generative Model
}

\author{
Emil Ballegaard $^{\mathrm{a}}$, Masashi Kajita ${ }^{\mathrm{a}}$, Paul Nicholas ${ }^{\mathrm{a}}$ \\ ${ }^{a}$ Royal Danish Academy, Institute of Architecture and Design
}

\begin{abstract}
This paper presents the development of a digital generative design tool for residential building that integrates qualitative data from potential users of buildings. The central aim is to understand and challenge the inherent biases in the design process of architecture for mobility impaired users, whose experiences might be difficult to understand for designers who often move around and use buildings without any difficulty.

Although Universal Design promotes designed environments that are more sensitized with the diverse difference of individuals, the most of design generating tools are based on empirically deducted human needs, objectifying the people or seeing them as useful in simply validating design ideas. There is a clear distance in between the real needs and wishes of wheelchair users and what architects imagine when designing.

Mixed-methods - expert interview, literature review and data analysis of disability blogs - are used to collect and analyse wheelchair users' experience. Accumulated qualitative data is, then, used as guiding input for the development of an explorative generative model that effectively produces large number of floor plans for residential architecture.

The developed generative model effectively selects floor plans that correspond with challenges described by the wheelchair users. The selected floor plans become informed starting points for spatial planning, which can guide architects to produce new and unexpected design solutions that are more sensitised to wheelchair users' experience.

The application of generative design tools in early stages of design tasks can help architects to understand users' needs and wishes, and thus challenge biased assumptions about wheelchair users' experiences. And yet, further research needs to be conducted in order to progress the system: additional user data and new design objectives can give rise to new hypothesis and allow the system to be more precise, responding to the complex reality of disabled people in their everyday lives.
\end{abstract}

Keywords. Mobility Impairment, Generative Design, Parametric Design, Qualitative Research

\section{Introduction}

There is an increasing emphasis on providing equal opportunities for all people regardless of age, ability or any other factors. The unjust nature of unequal access to the designed environment has, indeed, not gone unchallenged, though it is a slow process to make the built environment accessible and inclusive. Several design practices have emerged along the way, which promote designed environments that are more responsive to different personal conditions and circumstances. One of most significant is Universal 
Design (UD). In the United Nations Convention on the Rights of Persons with Disabilities (UNPRPD), devoted to its origin as introduced by Ronald Mace, UD is defined as "the design of products, environments, programmes and services to be usable by all people, to the greatest extent possible, without the need for adaptation or specialized design" [1].

The adoption of the concept in the UNPRPD consolidated UD's status as an internationally acknowledged concept; UD has since found its way into accessibility codes and design guides. The codified rules and guidelines aim to reduce inequalities in built environments by setting basic performance standards as the essential point of reference. And yet, those rules and standards are based on a quantitative approach which, not only reduces human bodies into average dimensions and ideal proportions, but also make it difficult to estimate the extent to which legislation can respond to the manifold interactions between diverse bodily variation and material environments [2]. This highlights a gap between two different approaches: one is quantitative, based on the standardised human body; the other is qualitative, based on the embodied experience of individuals.

In the effort of bridging this gap between quantitative and qualitative approaches to inclusive architectural design, this paper presents an attempt to develop a generative tool - developing architectural floorplans - which combines the requirements of building regulations with the statements of wheelchair users. The challenge is to investigate and overcome the inherent bias in architectural design against users whose experiences might be difficult to understand for designers (who often move around and use buildings without any difficulties). The paper specifically investigates how an evaluation of the generated floorplans can be done against the user statements and how a valid linkage can be provided with the architect as an interpreter between the qualitative statement and the architectural representation.

The project employs a mixed-method, combining both qualitative and quantitative approaches, that together forms a circular design process of data analysis, interpretation and design generation. The data is gathered through interviews, online data-extraction and literature reviews. The different data sources provide insights to the same issues relating to accessibility from regulatory, expert and user perspectives. This is done to elucidate the needs and wishes of wheelchair users from multiple perspectives. The generative system consists of three consecutive processes: 1) a generative system that produces thousands of floorplans; 2) a custom-made program that increases the level of detail in the generated floorplans. As a result of this, it is possible to extract measurements which relate to qualitative aspects of the architecture from the floorplans, and to use these to link them together with user statements; and 3) an evaluation system that allows the architect to search and pick the floorplan solutions which respond to different design requirements.

The use of generative systems to solve complex architectural design tasks goes back to the introduction of computation in architectural design. The paper contains a short review of recent academic and commercial projects by the architectural firm The Living and the real-estate company WeWork, both of which use generative design to solve multiobjective architectural design tasks with an emphasis on qualitative aspects.

The novelty of the tool and related methods lies in the preservation of qualitative data from users and experts which are used to inform a generative model. The aim of this approach is to avoid reduction of the qualitative content of the statements and to use a generative model to produce novel floorplan-solutions that respond to the concerns in 
the statements. The findings and discussions are based on a Master thesis conducted at the Royal Danish Academy.

\section{Background}

\subsection{Universal Design}

The term Universal Design (UD) can be misinterpreted as another word for accessibility. This is not entirely accurate, even though the two notions are intertwined. The term UD, coined by architect Ronald Mace in 1998, is a theoretical framework of designing for all users regardless of personal factors. UD is based on seven guiding principles that should be implemented to ensure accessibility and inclusivity [3]. UD is the most prominent among the practices which promote design that is responsive to different personal conditions and circumstances and is included in the UNPRPD. Accessibility is also included in the UNPRPD as one of the guiding principles. Article 9 of the Convention obligates State Parties to take appropriate measures to ensure that disabled people have access to facilities and services on an equal basis with others [1]. However, the general understanding of accessibility, defining the relation between user and context/society, has changed dramatically during the last century.

The notion originates from the field of war rehabilitation, with a medical- and pathology-founded understanding which focuses on the individual. This medical understanding was later replaced by a social understanding, which views accessibility as a complex relation between the individual and the material and social contexts within which they operate. Disability is thereby a social construction consisting of the interaction between the individual and its environments [4]. This understanding obliges society to minimise the exclusion of its citizens by definitions of standards and regulations. These rules and guidelines have undoubtedly reduced inequality caused by inaccessible architecture, yet they are regarded as generic regulatory pressures that many architects might consider to be restrictions rather than positive incentives. In addition, users are represented, in these rules and guidelines, by a certain type of standardized body - blind to the differences that human bodies possess [5].

Lately, this social understanding was replaced by a biopsychosocial understanding of accessibility. This recognises the interaction between the environment and the personal health condition with special focus on personal factors - humans are fundamentally different and a categorisation between persons with or without impairment is unnecessary and potentially exclusive [6]. UD had a strong influence in this shift towards the biopsychosocial understanding of accessibility. In theory, the inclusion of the UD principles in the UNCRPD is a safeguard of the personal factors inside the regulatory framework, but practical implementation is a difficult process: "The current codification of the principles of UD is being shaped by the rationale of administrative functions and, in part, by the language of construction industries and values of consumerism. Despite the advancement of the conceptual framework of disability, however, current codified rules and standards still draw attention to particular types of impairments" [7].

This indicates that personal factors are challenged in the practical application of rules and regulations, despite the implementation of design principles which aim to ensure precisely these individual needs and preferences. Other strategies are needed to 
ensure that general building requirements can be reconciled with individual preferences.

\subsection{Generative Design}

Generative design is defined as; "a goal-driven approach to design that uses automation to give designers and engineers better insight so they can make faster, more informed design decisions [8]." Generative design is often used as either a process of design optimisation or a process of design exploration, which is what this paper explores. Mattson describes; "The essential difference between design optimization and design exploration is the method for characterizing the outcome. Design optimization strategies have two distinct parts; formulate and converge. Here it is assumed that the problem can be formulated before the search and convergence begins. Design exploration strategies, on the other hand, are based on the belief that the problem formulation evolves during the process of searching and converging, thus ultimately leading to a more informed optimal solution. In this way, design exploration is both divergent and convergent [9]."

According to Krish [10], generative design processes are composed of three components: 1) a design schema; 2) a means of creating variations; and 3) a means of selecting desirable outcomes. Differing descriptions of these components can be found, and they can be broken down into further processes according to the aim of the designer. The generative design process presented in this paper, iterates through five steps: 1) the definition of design objectives and constraints; 2) the construction and tuning of a generative system; 3) the generation of designs; 4) the evaluation of designs; and 5) the selection of design(s).

As a digital system can a generative design model only be informed by quantitative data, in the form of numerical values. Consequently, a generative design process which aims for qualitative aspects of the design, is dependent upon a strategy of quantification and a predefined system of data management to ensure a coherent correlation between the qualitative input and the generated design. As the reference projects presented in this paper show, there are several approaches to quantify qualitative data or qualitative assumptions. Bernholtz and Fosburg developed a generative program called $L O K A T$ in 1971 to solve multi-dimensional design problems related to space planning due to the fact that, "...the designer alone cannot effectively deal with the complexity of the problems currently facing him" [11]. The solution presented to overcome this problem is a floorplan generating system that is informed by relationship values. Based on intuition and empirical experience of the designer, the relationship values describe the importance of relations between certain rooms; these are used to make decision tables which define the acceptable trade-offs between different interests in a planning situation. The qualitative information that informed this early computational project of architectural planning is the preferred adjacencies between rooms which are represented as numbers.

Within the last decade, companies like WeWork and The Living have applied generative strategies in both research and commercial work, integrating user perspectives in partially qualitative manners. WeWork build shared workspaces for rental worldwide in more than 800 office buildings. The scale of the company enables WeWork to collect a substantial amount of data from their rental statistics and a massive amount of BIMinformation from their office spaces. Bailey et al. [12] collected 56 variables to describe the qualities of the office spaces based on inherent rule of thumb among architects, previous studies and interviews. Machine learning was applied to find patterns in the 
variables, and the data from customers and buildings of WeWork, with the purpose of finding relationships between the behaviour of renters and the layout of office spaces. The relationships are used to predict the commercial success of these office spaces, and to help the architects to "create desirable offices that are constantly leased..." [12]. Anderson et al. [13], also affiliated with WeWork, describe the development of procedural algorithms to generate floorplans for office layouts. The study aims to augment the architect's decision-making process by the automation of tedious design tasks, in this case desk-layouts in the office spaces owned by WeWork.

The success of the algorithm is determined by the efficiency measure (the ratio between desk count and area of the office) of the auto-generated layouts, compared against the actual offices of WeWork. In $77 \%$ of the cases, the algorithm matched the efficiency of the architects and in 6\% it managed to increase the efficiency of the office space. The authors speculate on a merge of the results from the two studies and state; "As our previous research has shown [13], it is possible to have a machine learning complex design patterns for the evaluation of design, and now potentially for the laying out of furniture" [12].

Nagy et al. [14], from The Living, describe the development of a generative design methodology consisting of a generative system, a process of design evaluation and a method to search across the generated designs based on the evaluations. The generative model produces thousands of floorplan layouts for the software company Autodesk's office building in Toronto using a custom-made algorithm. The performance of the designs is measured by six discrete evaluation metrics, (adjacency, work style, distraction, productivity, daylight and views to outside), which consist of user preferences, simulations and simple measurements of the floorplans.

Ordinal-scaled surveys are conducted in order to gain an understanding of the preferences among the Autodesk workers according to workstyle (ambient and distraction level), and adjacency (preferred neighbours and amnesties) [15]. Simulations of daylight and productive congestion [16] are carried out on each floorplan to provide insight to some of the dynamic elements of an office space. The surveys, simulations and other measurements (such as distance between employees), result in quantitative data that describe the performance of each floorplan. A multi-objective genetic algorithm is used to search through the floorplan solutions, based on the performance metrics, and enables the architect to find floorplans that perform well according to interest.

As the references show, generative design has been used with the intention to include qualitative aspects of users in computational design for decades. The basic premise of quantification is largely unchanged, despite the fact that new tools are developed to give insight to complex relations from numerical values. The quantitative data can reveal relations and correlations, but it is still disconnected from the meanings and quality of experience that qualitative data are able to contain. In what follows, this paper presents a method that seeks to include personal factors, described by qualitative data, in an explorative generative design process. The aim is to overcome the regulatory challenges presented by the subjective and contextually bound preferences described. The method uses quantification to generate metadata that can inform the generative system, but preserves the original data for evaluation. 


\section{Method}

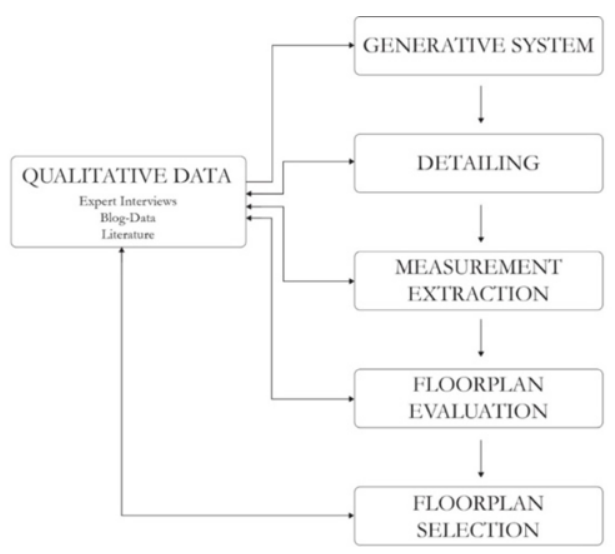

Figure 1. Diagram explaining the relation between the qualitative data and the design generation.

The methodology presented in this paper is a mixed-method: a combination of several complementary sub-methods which are for either data processing, or for generating and evaluating floorplans of residential architecture in a digital framework [FIG. 1]. The mixed-method allows the effective evaluation of floorplans against the user statements and vice versa, leading to the production of novel floorplan solutions that meet the demands of both users and regulations [17]. The methodology has been developed and tested within a research approach primarily based on abductive reasoning and heuristics. It can be argued that these approaches leave more room for creativity than pure deduction, whilst still operating within some predefined structure (such as building regulation and user preferences). The heuristic approach is an advantage in a design processes like this, in which the main objective is to create novel floorplan solutions suitable for wheelchair users, but the specific design is unknown.

User-data and data management are central parts of the methodology. The three primary data sources in the project are expert interviews, blog data, and relevant literature. Each data source brings different kinds of perspectives, has a different agenda and, therefore, underpins the different purposes of the project. The variety of data sources allows the project to avoid unnecessary content reduction of the qualitative data through quantification, and unjustified qualitative assumptions based on numerical information. Expert interviews and scientific literature inform the generative system prior to the first floorplan generations and are afterwards used as a consultant through the evaluation process. The blog-data is used as a fictive client, or potential user, whose preferences and opinions are found through text search and reviews. The following sub-sections describe each method used in the project in chorological order.

\subsection{Interviews}

Two expert interviews were conducted to gather knowledge from two expert groups. A wheelchair user, who represents disability organisations ${ }^{1}$, was interviewed first. This was

${ }^{1}$ RYK - Rygmarvsskadede i Danmark 
to gain experience-based knowledge of accessibility. Following this, an interview with industry professionals, who work with accessibility, was conducted ${ }^{2}$. To guide the conversation, and to ensure a valid connection between theoretical concepts and the interviewees' experience, a concept of operationalisation [18] was applied and visual material were prepared prior to each interview. The visual materials, including references, architectural drawings and images, were used to make sure that the areas of interest were understandable through specific examples. As a data source, the expert interviews are characterized by highly professional and analytical considerations, giving access to relevant information on several levels. The experts were able to convey important information regarding both regulatory and administrative issues, such as complaints about inaccessibility and concrete design suggestions, to solve seemingly everyday challenges. For example, experts interviewed highlighted the fact that visual overview of the home is important to many wheelchair users, due to several social and safety reasons; this information become one of the central topics of this study.

\subsection{Data mining}

Blog-data is extracted from various internet-based blogs in an online community who write about living with mobility impairment ${ }^{3}$. The blogs consist of personal statements, and the consideration and content of the blogposts is highly varied. Almost everything, from cooking to fashion and politics, is discussed in these blogs, but generally with at least an underlying, or sometimes very pronounced, focus on accessibility, often posed as the object of critical discussions and concerns. One blogger, for example, describes how their accessible hotel room wasn't accessible at all due to the width of the door, and the struggle they had to go through to get an equitable room.

The low reliability, personal content, and unstructured nature of blogposts necessitate large amounts of data to answer specific questions in a later design process. To include this extensive amount of data, the blogposts are collected in text documents from which further search can be performed. Inspired by software designed for qualitative research such as Nvivo, and in order to integrate the relevant data into the design process inside the same computational framework, a text search tool was developed in Rhinoceros/Grasshopper. The text search tool is used to search for user considerations about architectural challenges that are associated with specific living spaces, objects, or features through a comparison of the user statements against the floorplan sketches. In order to reduce the extent of irrelevant search results in the dataset, the text search method is built on a metasearch technique that requires multiple words in the same sentence. For example, it could be a combination of an architectural object such as "door" and a descriptive word such as "wide". Once this search criterion is met, a data package is created with the sentence content, location and source, making it easier to handle fragments of a large dataset in a dynamic design process.

The blogs contain information created on the user's own initiative, without any prior questions, as a stream-of-consciousness; this gives the blog-data a high degree of ecological validity [19]. Open and accessible spaces, outlook, and privacy are some of the most mentioned and desired architectural qualities found in the blog-data that have been central in the following design process. The considerations and preferences found

\footnotetext{
${ }^{2}$ Dansk Handicap Forbund

${ }^{3}$ These include: http://handicapthis.com/blog/, https://www.thebimblers.com/, http://www.thesqueakywheelchairblog.com/, https://www.simplyemma.co.uk/.
} 
in the blog-data are unstructured and sometimes contradictory, which makes it difficult to use the blogposts as direct design instructions but are highly important as bases for design-trade-off discussions. The level of personal involvement found in the blog-data highlights other facets of what is described by both experts and relevant literature. The experienced trivialities, preferences and daily challenges are mainly explained in an everyday context, and with a pronounced personal focus, which makes the experiences more understandable for able-bodied readers.

\subsection{Literature reviews}

Literature reviews of scientific- and professional literature are the project's third source of data and consist primarily of regulations and recommendations from governmental organizations and NGOs. The Danish building regulation (BR18) [20] have been studied, and requirements as numerical values is extracted for the purpose of guiding the generative design process. The literature, including BR18 describes a number of recommendations and mandatory legal requirements that have been used in the definition of design evaluation criteria. The building references found in the literature as examples have been an important inspiration in selecting the constituents of the floorplans and the internal room connectivity.

\subsection{Development and tuning of a generative system}

The generative system developed for this project is scripted in Grasshopper and is based on Egor Garilov's Grasshopper-plugin Magnetizing Floor Plan Generator (MFPG) [21]. MFPG is a program developed to generate floor plans for public buildings; such places often serve multiple functions, making the design process complex (and often contradictory) due to the large number of design objectives [22]. In this project, the program is used to create simple diagrammatic representations of residential architecture in the form of floorplans which consist of rectangular rooms, with lines between them representing the connection between the rooms. MFPG is based on a rule-based iterative algorithm which uses a spatial connectivity diagram as input. Spatial connectivity diagrams are born of Space Syntax theory; they describe the number of rooms, their size and interconnections as node diagrams [23] [FIG. 2]. Prior to generation, the algorithm is set according to the target, time consumption and precision.
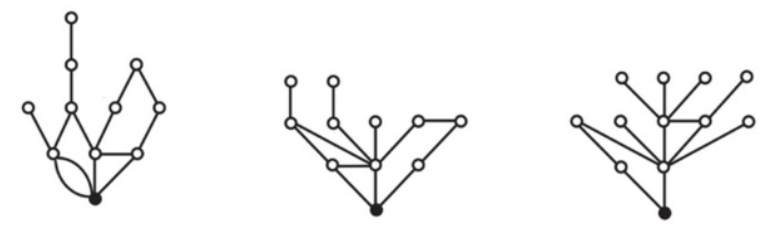

Figure 2. Three connectivity diagrams representing 3 different French houses by Bill Hillier [23].

A custom-made program is developed to increase the level of detail of the generated floorplans. The program is based on a principle of randomness within defined frameworks. One example of this principle can be found in the positions of windows; the ratio of wall/window is defined for each room, but the specific position of the windows, their preferred direction and likewise preferences is not. The program generates hidden 
sub-geometry as points and lines which are used to extract measurements for later evaluation of the floorplans.

\subsection{Measurements and mapping}

The generated floorplans are measured for three reasons: 1) to compare the designs against each other; 2) to evaluate the floorplans based on non-intuitive measurements, and 3 ) to be able to explore a great number of designs than would be possible to inspect manually [24].

The selection of measurements is a process through which some of the issues identified in the data are related to measurable entities and subsequently corresponding methods of measure are found. For example, if a user complains about the distance from the front door to the bathroom, a measure of this distance could be taken in all the generated floorplans. This measure would probably not lead the architect to the perfect solution, but it would relate to the problem stated by the user. The numerical values are not intended to be used to classify the floorplans based on normative parameters, such as either good or bad, but used rather as indicators. The idea behind the use of numerical values as indicators is to combine several measurements to target specific architectural preferences or challenges stated among the users. An example of such a combination of measurements could be: 1) a measure of the area visible from the entrance, 2) a measure that explains the shape of the living room, and 3) a measure of the distance from the entrance to kitchen. The combination of these measurements may indicate whether the layout of the floorplan could potentially solve specific challenges or preferences of the intended user(s) related to the entrance of the house.

The measurements extracted from the floorplans are remapped in a range between 0 and 1, and a method of searching for floorplans of interest and visual evaluation of the floorplans is developed based on a three-dimensional coordinate system [FIG. 3]. The remapping of the numbers allows all the measurements to be evaluated inside the same coordinate system. Three of the extracted measurements, or a combination of several measurements, are selected as $\mathrm{x}, \mathrm{y}$ and $\mathrm{z}$ values respectively, thus forming a coordinate point for each floorplan. The position of the point in the coordinate system maps all of the floorplans in relation to the selected values, (this could be the entrance example described above). The combination of measurements can be continuously altered or combined according to the focus of the architect.

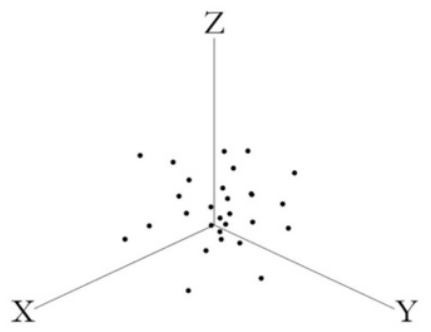

Figure 3. A 3-dimensional coordinate system with 27 floorplans represented as points.

Some measures are binary and describe whether or not a particular feature, such as a window, is present, while other describe an area, distance or ratio. It is important to understand what the numbers describe and to know the range in which they occur to use 
mathematical operations that give the right measurement the correct weight (relative importance) in a comparison across the floor plans.

\subsection{K-mean clustering}

$\mathrm{K}$-mean clustering is a vector quantification method that finds $\mathrm{x}$ numbers of mean values in larger amounts of data and defines clusters from the data points closest to the mean values. If we use the entrance example again, each cluster would contain solutions with specific characteristics according to the measurements used to find floorplans that responds to the needs and preferences related to the entrance. The mean values, known as cluster representatives, represent the average of the cluster [FIG. 4]. The cluster representatives provide important information about where floorplans with specific characteristics can be found in the coordinate system. The representatives are used to navigate among the floorplans in the process of searching for an instance that targets specific architectural qualities found important among the users. In practice, the k-mean clustering feature of Mateusz Zwierzycki's Grasshopper plugin $O w l$ [25] was integrated into a custom-made Grasshopper script, which allows the architect to freely combine extracted measurements and visually evaluate the floorplans of interest.

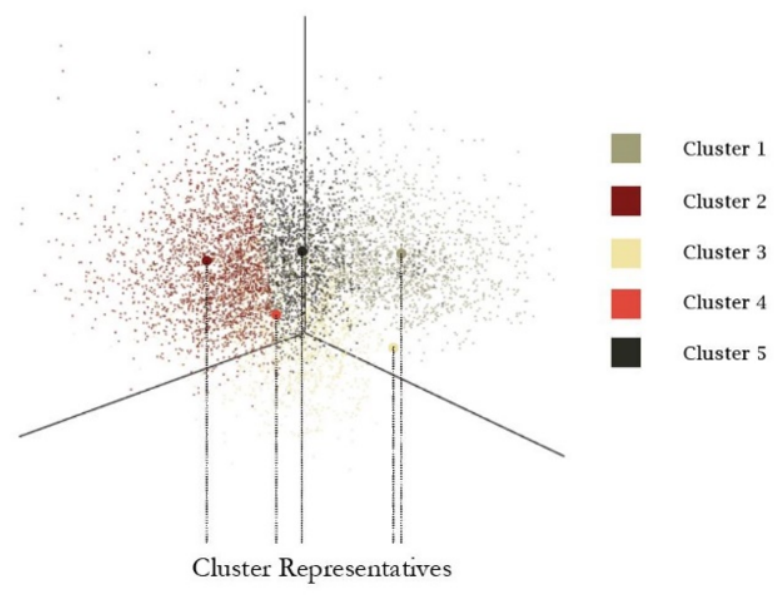

Figure 4. 100,000 floorplans, divided into clusters using K-mean clustering method.

The above-described methods allow the architect to generate a substantial number of floorplans and find the architectural designs that corresponds to the preferences of the users. The qualitative data from users is preserved, and measures from the generated floorplans are evaluated against the data. Quantifications and generalisations are made to inform the generative process; the numerical values guide the search across the designs but are not used as a basis for the final selection of the floorplans. The method positions the architect in a relation between the roles of system developer, data analyst, and design interpreter. The method could be criticised for its subjectivity and the time-consuming processes of system-setup and interpretations. We argue that an in-depth understanding of the generative system is crucial in order to find suitable solutions, and that a preservation of the qualitative data is needed to overcome the problems of individuality in the regulatory framework described. 


\section{Development Process and Result}

All the floorplans generated employing the above-mentioned methods, are equally sized and consist of approximately 100 square meters. Each includes an entrance, a living room, a kitchen, a bedroom and two bathrooms. In addition is there a so-called ramp-room which connects the less private areas (entrance, living room and kitchen) with the bedroom. The size of the dwellings is a little below average for single family homes and a little above average of townhouses in Denmark [26]. The types of rooms included are also fairly average, based on personal experiences, with the exception of the ramp-room and the extra bathroom. References such as Rampenhaus ${ }^{4}$ shows that a ramp can provide access and add spatial qualities when well-designed. Following this, the ramp-room is used as a driver for novel floorplan solutions in the generative process, even though this architectural element might not be suitable for all users. The ramp-room could have been a different room or element and can easily be left out in another design generation. The additional bathroom is included due to challenges related to awkward and embarrassing situations described in the blog-data, which might be possible to solve with both a private bathroom and a bathroom for guests.

The order of, and connectivity between, the rooms are central constraints in the generative process and have a huge impact on the layout of the floorplans. The spatial connectivity diagrams are used to both analyse the connectivity in state-of-art examples ${ }^{5}$ of accessible architecture, and to sketch spatial configuration for the generative process. A significantly higher diversity among the generated floorplans was found using relatively linear spatial connectivity diagrams, in contrast to interconnected and dense diagrams (see FIG. 2 and FIG. 5) in which not only the connectivity, but also the position of the room, is quite predefined. Given that the aim of this project is to explore new solutions, the diagram chosen for the generative process employs linear spatial connectivity [FIG. 5].

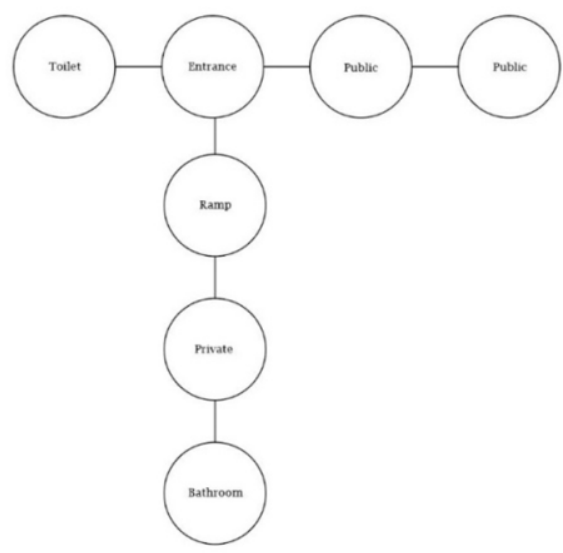

Figure 5. The linear spatial connectivity diagram used for the generation of the floorplans.

\footnotetext{
${ }^{4}$ Rampenhaus, Florian Höfer Architekt, Gstadt am Chiemsee, Germany, 2004

${ }^{5}$ These include: Laurent House, Frank Lloyd Wright, Illinois, United States, 1951; Maison Bordeaux, OMA, Bordeaux, France, 1998; Villa Deys, Paul De Ruiter, Rhenen, The Netherlands, 2001
} 
The algorithm used in the generative system is set according to the spatial connectivity diagram, and the balance of the generation-speed and precision of the generation according to the defined constraints. The explorative agenda of the project requires that the precision (objectives and constraints) of the system should be defined loosely enough that new solutions can be generated, but adequately tight to ensure that the majority of solutions can be used as a basis for further design development. The output of the generative system is thousands of floorplans consisting of rectangular, named rooms and lines that illustrate the access between the rooms [FIG. 6].

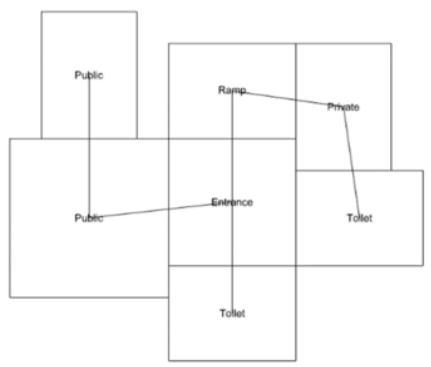

Figure 6. Floorplan type 3990.

The output of the generative process is rather diagrammatical. Information about qualitative aspects of the architectural features which can reasonably be said to relate to the simple floorplans is searched for across the data sets. One of the experts interviewed stated that the kitchen is one of the most dangerous places in an average home and it is not an exception for wheelchair users. This could be, for example, because wheelchair users often use their lap to transport groceries, utensils, and so forth. It was also stated that, rooms for socialisation (mentioned as public in the diagram) that hinder the ability of wheelchair users to move around freely, are examples of exclusive design, highlighting the inequality between able-bodied users and wheelchair users. Several bloggers confirmed this and expressed their contentment with large, open kitchens and social rooms in which they were able to move around unhindered. The programme of the floorplans is modified based on these statements: to make space for free movement, and to possibly reduce the dangers of the kitchen, the entrance, the living room and the kitchen are merged into one large room [FIG. 7].

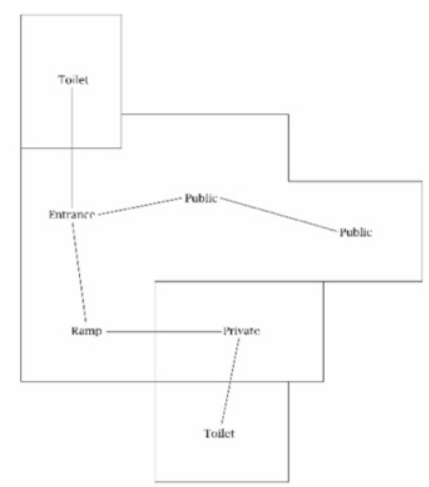

Figure 7. Floorplan type 9075 with a large room for socialisation and private bathrooms and bedroom. 
A custom-made program is developed to increase the level of detail in the floorplan and thereby enable a more qualified evaluation of the floorplans against the data. Wall thickness, doors, windows and interior elements are added to the generated floorplans; these additions give the floorplan a level of detail similar to floorplans found in real estate advertisement. This higher level of detail enables the architect to have meaningful conversations with laymen about the layout of the floorplan and enables important measurements, such as distances between the various functional areas of the house, to be found; this gives insight as to how the individual floorplan meets the demands of the users [FIG. 8].

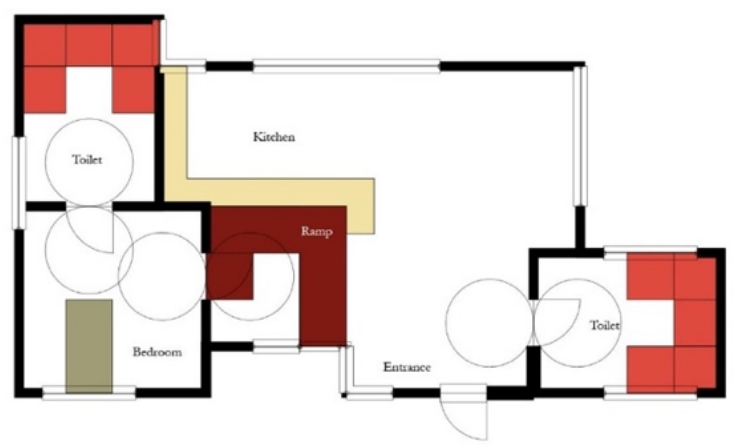

Figure 8. Floorplan type 709 - with increased degree of detail and specified interior features.

As an evaluation framework for the thousands of floorplans which can address the challenges described in the data, 31 initial measurements are extracted from each floorplan. These include areas, distances, dimensions and quantities while other measurements aim at describing qualitative architectural aspects, such as overview, outlook, shapes, potential backlight and so forth. Outlook is a common quality in architecture and is also considered very important among the bloggers. Outlook can inform the resident of the exterior conditions as they relate to the interior situation. For example, if one hears a police siren or car alarm, wants to check on the weather, or simply desires to look out the window. Mobility impaired users often spend more time in sedentary or lying positions and it helps to position windows and doors to provide good sightlines throughout the space.

Overview relates to visual accessibility and the organisational legibility of a room from certain positions in space. Overview is found important in the literature and among the experts interviewed. Being able to read and understand our environment whilst unobstructed, either visibly or physically, is essential for people in general and in particular to some who are mobility impaired. Seven of the 31 measurements relating to overview are extracted from each floorplan. Isovist Sampling is used to measure the area visible from a given vantage point in space and this is used to assess overview with metric measurements [FIG. 9a, 9b, 9c]. It is important to state that the overview, as well as the outlook, of an occupant at the given point in space contains a perceptual complexity that cannot be reduced to a single measurement. The value can therefore not be used to describe the experience, but it can provide useful information about the material context from which a user experience might emerge. The Isovist Sampling provides a numerical understanding of geometric relations related to the visual perception of a potential user, but the technique is unable to convey experiential meanings. 

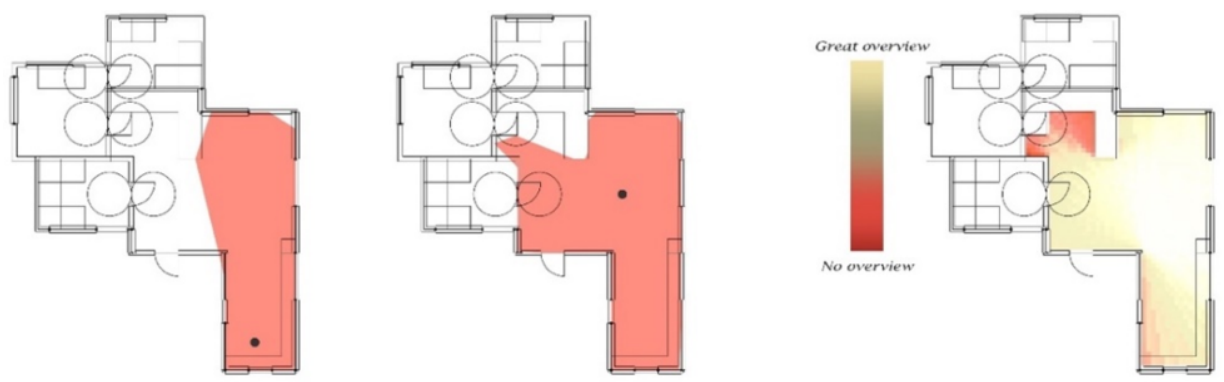

Figure 9. a) a position in the house with a low degree of overview. b) a position in the house with a high degree of overview. c) a false colour map showing the measured overview across the large social room.

In this project, outlook and overview are used as guiding principles for case studies of searches for specific architectural qualities across the generated floorplans. In the case presented below is three of the 31 measurements chosen for evaluation of the floorplans and subsequently to guide the corresponding search across floorplans. The measurements are chosen in this case due to their relation to outlook and overview of a potential user. The measures are: 1) the shape of the house; 2 ) the average of visible window area from 1500 points in space inside the large social room; and 3) the range of visible area from the same 1500 points. The measurements of each floorplan are represented through a point according to the method described in 3.5 and K-mean clustering is applied. A division of the evaluated floorplans into five clusters is found to be suitable to balance an intuitive understanding of the differences between the floorplans and the diversity among the cluster representatives.

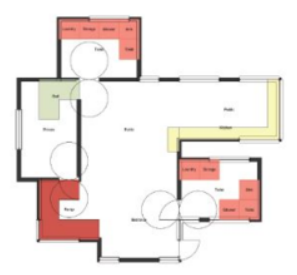

Cluster Representative 1: Model 6502

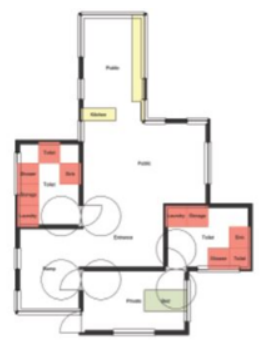

Cluster Representative 2: Model 8409
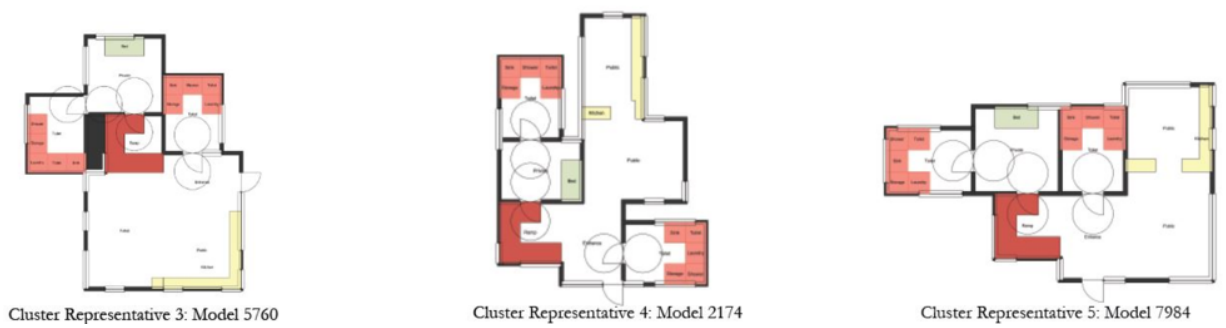

Figure 10. The 5 Cluster representatives found in a search using the measurements described. 
The cluster representatives [FIG. 10] indicate the differences between the floorplans found in the various clusters related to the three measurements used. Cluster representatives 2, 3 and 5 show a high average of window area visible across the house, and especially number 3 and 5 have a large social room connected with the entrance to the house. The kitchen in number 3 is situated in the social room, whereas the kitchen of number 5 is placed as an adjacent room in connection with the large social room. The range of visible area in the social rooms of number 3 and 5 is low, due to the openness of the room and the absence of small spaces inside the room; this corresponds favourably with statements from both the experts and the bloggers. The findings suggest that the room composition of number 5 provides better opportunities to divide the spaces and establish more private zones inside the social room, responding to the quality sought after among many of the bloggers. Accordingly, the project continued a search for suitable floorplans that correspond to user statements inside cluster 5 .

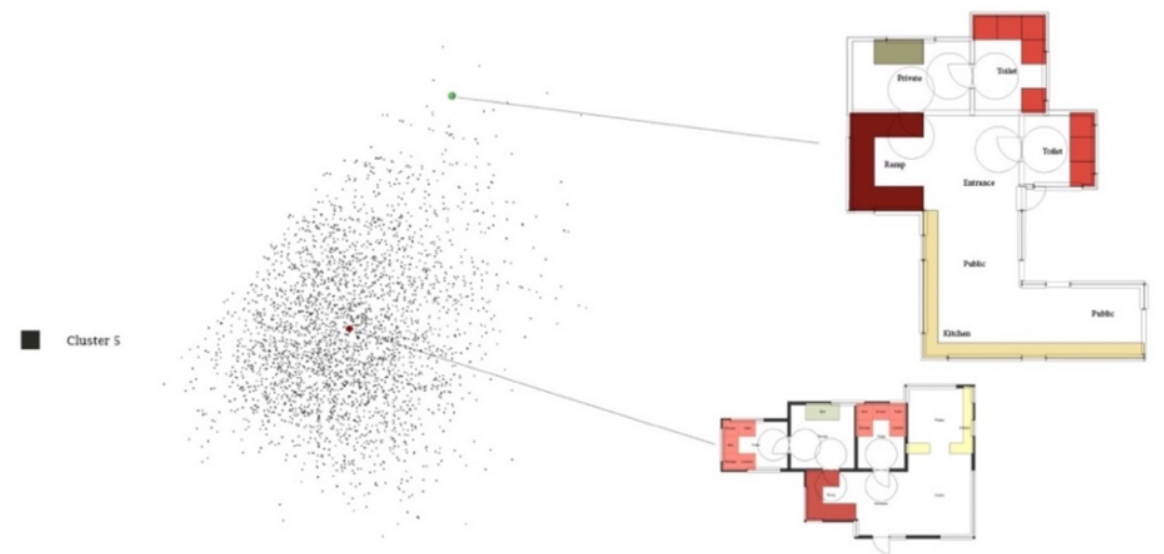

Figure 11. Cluster 5 and the cluster representative, floorplan type 7984 (bottom) and floorplan type 3159 found in the border zone between cluster 5 and cluster 3 (top).

The blog-data is used as a fictive user to guide the selection of floorplans for further design. In order to do this, wheelchair-user statements which relate to architectural elements (as described in 3.2) are found and read by the architects as challenges to the selected floorplan. User statements such as: "As we opened the door to our accessible cabin, we were relieved to see it was a large space" guide the search across the floorplans. The search leads the project in the direction of the border zone between cluster 5 and 3 , where a number of floorplans combine the described qualities with a large kitchen, and areas of the room for socialisation that could be divided to create intermediary between a private and a social room [FIG. 11]. Several of the floorplans correspond to the user statements but floorplan number 3159 is chosen. Following the methods described, this particular floorplan was found to be the best solution to meet both user preferences and building regulations. The selected floorplan can easily be exported from the generative system to a common computational design framework for further design [FIG. 12]. Architects can use this augmented decision-making tool to find solutions that are unforeseen and that comply with both the user preferences and the regulations. We believe that this offers an opportunity to reduce the biases found in architectural design and to avoid the mere replication of existing design solutions. 


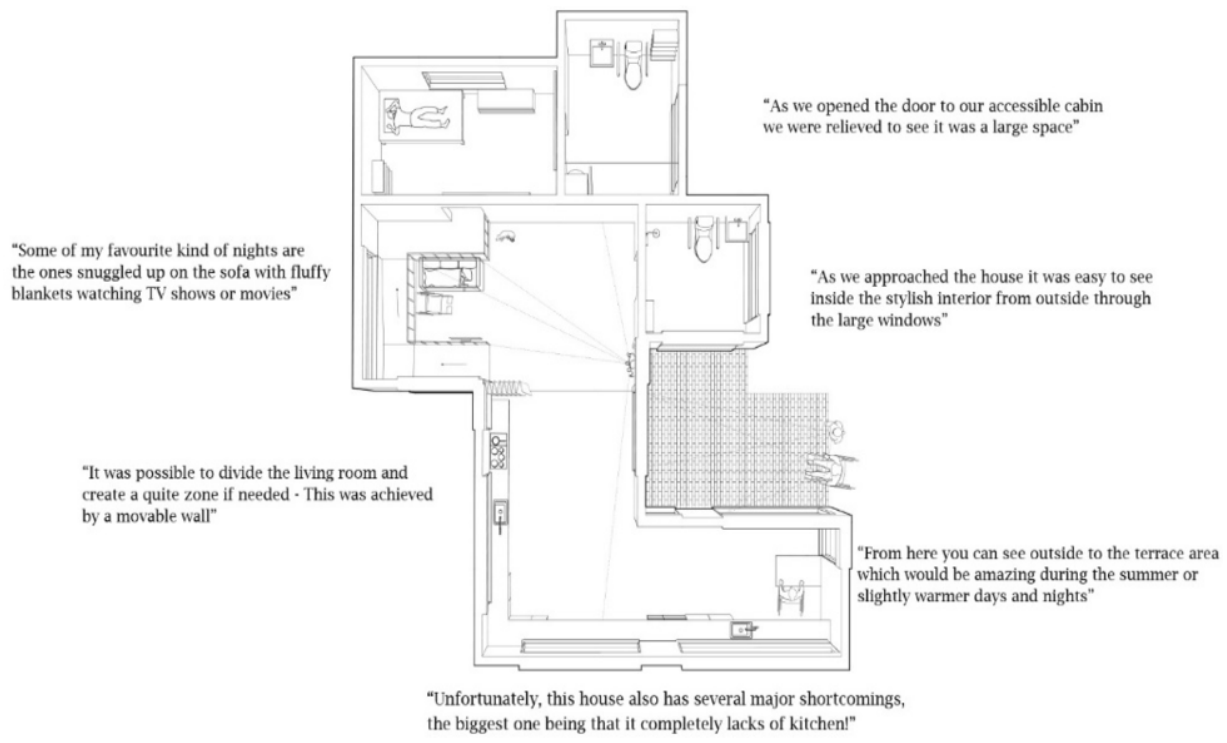

Figure 12. A visualisation of floorplan type 3159 surrounded by some of the statements that are used to pick the specific floorplan.

\section{Discussion}

Floorplan number 3159 complies with the included building regulations and our understanding of user preferences but does not necessarily represent the greatest architectural solution for the targeted user. Our intention is to present the preliminary method and its qualitative and quantitative sub-processes through their application, which, in the case presented, has led to the selection of floorplan number 3159. This method described depends largely on the subjective judgement of the architect and does therefore not provide the same objectivity as other generative design projects. However, we believe that the selected floorplan responds to the user statement included in the evaluation process [FIG. 12]. Floorplan number 3159 contains a ramp which also creates a niche - in the layout of Figure 12, this is used as a TV-corner connected to the social room. The social room is easy to divide and thereby separate the entrance, the toilet and the ramp/niche from the kitchen. There is a clear overview from the front door, the entrance to the house is combined with a small courtyard due to the shape of the house, and the position of the windows gives the guest or homecoming-resident a glimpse of the activities in the social room. The windows also provide the residents with a good outlook to the courtyard/entrance. The house is not lacking in kitchen space, and the large table allows the kitchen to be used for many different activities and for social cooking.

Projects from The Living and WeWork are presented in the background section as reference examples that have succeeded to include user data in a generative process. The 
aim of WeWork is to understand the preferences of their renters in order to design office spaces that match them, thereby increasing the profit of the company. To do so, they compare rental statistics with numerical descriptions of the offices that are rented out and the qualitative level of the input data is therefore low. The Living use ordinal-scaled surveys as a data collection method to understand the user preferences according to a number of variables. The survey is constructed of questions to which the user responds with numbers that are believed to express their preferences. The user data becomes quantitative but refers to qualitative aspects of the office space experiences.

This paper has presented the initial development of a new method of floorplan generation, based on qualitative information from wheelchair users, in order to propose novel architectural solutions that respect the physical conditions of the users, the personal preferences, and the building regulations. In pursuit of this aim, the qualitative data used in the project is characterised by high ecological validity and a subsequently low reliability which means that highly subjective aspects can be included; this, however, makes mathematical comparisons much more complicated. In contrast, the data used by WeWork and the Living is characterised by a higher reliability which enables statistical operations that can widen the scope of the investigation and save time by lowering the required level of manual data interpretation. Despite the advantages of the quantitative methods, the research findings show that the preservation of the qualitative data and its use in the evaluation of the generated floorplan is necessary to maintain the qualitative user perspective throughout the digital processes.

\section{Conclusion}

The explorative generative process described in this paper produces thousands of floorplans. Through the methods for evaluation and search, we were able to find novel and unforeseen floorplan layouts that, we believe, respond to the user statements and to the framework of building regulations. The methods and sub-processes are in an early stage of development and need much more work before being applied in a non-fictive design process. However, based on the preliminary results, we believe that the integration of qualitative and quantitative methods into generative design could be a way to reconcile personal preferences with building requirements.

Due to the early stage of development, we are not able to draw any justified conclusions about the inherent biases in architectural design for wheelchair users based on the generated floorplans. However, through the interviews and the extensive online data-extraction we can conclude that the problem exists. To produce better architectural designs, and to gain a deeper understanding of these biases, it is necessary to develop the generative tool in several ways. As stated, we believe that the preservation of the subjective aspects of the data is important to target the aims described. Yet, we also acknowledge that quantitative methods hold a significant potential to improve the tool.

An inclusion of methods that can extract relevant metadata to the qualitative data, such as the Universal Dependencies [27] would be a productive next step in combination with an improved generative model. Many more measurements extracted from the floorplans are needed to link even more specific and personal preferences with the generated designs, and finally, a user-friendly interface is needed before the tool can be tested by other architects and designers. 


\section{References}

[1] United Nations, General Assembly, Convention on the Rights of Persons with Disabilities and Optional Protocol, United Nations, New York. 2007.

[2] Imrie, R, "Universalism, universal design and equitable access to the built environment", Disability and Rehabilitation 34(10) (2012), 873-82.

[3] Conell, C.R. et al., The Principles of Universal Design (1997), https://projects.ncsu.edu/ncsu/design/cud/about_ud/udprinciplestext.htm.

[4] Goldsmith, S. and PRP Architects (Firm), Universal Design: A manual of practical guidance for architects, Architectural Press, Oxford, Boston, 2000.

[5] Kajita, M, "Inclusion in Danish architectural education and design process", Advances in Design for Inclusion: Proceedings of the AHFE 2019 International Conference on Design for Inclusion and the AHFE (2019), 3-14.

[6] WHO, International Classification of Functioning, Disability and Health: ICF, World Health Organization, Geneva, 2001.

[7] Kajita, M, "Re-addressing the social dimension of accessibility: towards an inclusive society", Form til Velfoerd/ Forming Welfare, The Danish Architectural Press, Copenhagen (2017), 280-291.

[8] Autodesk, Demystifying Generative Design: For Architecture, Engineering and Construction (2019), https://damassets.autodesk.net/content/dam/autodesk/www/solutions/generative-design/autodesk-aecgenerative-design-ebook.pdf.

[9] Bringham Young Universitys Design Research Group; What is Design Exploration? (2014), https://design.byu.edu/blog/what-design-exploration-0

[10] Krish, S, "A practical generative design method", Computer-Aided Design 43(1) (2011) 88-100

[11] Bernholz, A., Fosburg, S., "A Generalised Program for Transforming Relationship Values into Plan Layout", Advanced Computer Graphics - Economics Techniques and Applications (1971), 449-463.

[12] Anderson, C., Bailey, C., Heumann, A, et al., "Augmented space planning: Using procedural generation to automate desk layouts", International Journal of Architectural Computation, 16 (2018), 164-177.

[13] Bailey, C., Phelan, N., Cosgrove, A., et al., "This Room Is Too Dark and the Shape Is Too Long: Quantifying Architectural Design to Predict Successful Spaces”, Humanizing Digital Reality (2017), 337348.

[14] Nagy, D., Lau, D., Locke, J., et al., Project Discover: An Application of Generative Design for Architectural Space Planning, SimAud 2017 (2017), 59-67

[15] Villaggi, L., Stoddart, J., Nagy, D., et al., Survey-Based Simulation of User Satisfaction for Generative Design in Architecture, Humanizing Digital Reality (2017), 417-430

[16] Nagy, D., Villaggi, L., Stoddart, J., et al.; The Buzz Metric: A Graph-based Method for Quantifying Productive Congestion in Generative Space Planning for Architecture, Technology/Architecture + Design 1 (2) (2017), 186-195.

[17] Groat, N., Wand, D., Architectural Research Methods, Wiley, New Jersey, 2013.

[18] Køppe, S., En moderat eklektisme, Psyke \& Logos 29 (2008) 15-35.

[19] Helles, R., Køppe, S., "Kvalitative metoder", Humanistisk videnskabsteori, Lindhardt og Ringhof, Copenhagen (2014) 535-575

[20] https://bygningsreglementet.dk/

[21] https://www.food4rhino.com/app/magnetizing-floor-plan-generator

[22] Garilov, E. et al., "Computer-aided approach to public buildings floor plan generation: Magnetizing Floor Plan Generator", $1^{\text {st }}$ International Conference on Optimization-Driven Architectural Design (2019).

[23] Hillier, B., Space is the machine, UCL, London, 2007

[24] Nagy, D., Designing measures, Autodesk, New York, 2017. https://medium.com/generative-design/designing-measures-2c66a71b2ff3<

[25] https://www.food4rhino.com/app/owl

[26] Danmarks Statistisk (Statistics Denmark): BOL103, 2019.

[27] De Marnefee, M. et al., Generating Typed Dependency Parses from Phrase Structure Parses, Proceedings of the Fifth International Conference on Language Resources and Evaluation (2006). 